\title{
The usefulness of the transesophageal Doppler in the anesthetic management of Morgagni hernia repair - $A$ case report
}

\author{
Nuno Morais de Babo, Maria Teresa Monteiro $\square$, Ana Bernardino Santos $\square$, \\ Cludia Raquel Carreira \\ Authors' affiliation: \\ Praceta Prof. Mota Pinto 3004-561 Coimbra. Portugal. \\ Correspondence: Nuno Miguel Morais de Babo, Department of Anesthesiology, Centro Hospitalar e Universitário de Coimbra, \\ Coimbra, Portugal; Phone: 916240296; Mobile: 351 916240296; E-mail: babo.nuno@gmail.com
}

\section{Abstract}

Morgagni hernia in adults is a rare condition and is associated with complications such as recurrent chest infections, respiratory failure and cardiac tamponade. Its correction represents an anesthetic challenge. Intraoperative esophageal Doppler provides important information in real time about the patient's cardiac function and vascular filling, aiding in decision making by the anesthesiologist. We report a case of a 70-year-old woman, ASA Physical Status-III, and presented with chest pain, dyspnea and hypoxemia. Her chest x-ray revealed opacification of $2 / 3$ of the left hemithorax and $1 / 2$ of the right. Computed tomography confirmed a bilateral Morgagni hernia. The patient underwent immediate corrective surgery. Intraoperative cardiovascular function was monitored with esophageal Doppler. Compression and decompression of thoracic structures produces enormous hemodynamic and respiratory impact. After herniated contents removal it showed an increase in systolic volume and cardiac index and a correction of flow time. Transient arterial hypotension was verified, requiring fluid therapy and vasopressor support. Diaphragmatic defect was corrected and general hemodynamic stabilization was achieved. Patient was discharged asymptomatic. The esophageal Doppler was important in this case because it allowed us to measure fundamental hemodynamic variables in real time, such as cardiac index or systolic volume, and to subsequently act accordingly.

Key words: Esophageal; Doppler; Morgagni hernia; Anesthesia

Citation: Babo NM, Monteiro MT, Santos AB, Carreira CR. The usefulness of the transesophageal Doppler in the anesthetic management of Morgagni hernia repair - A case report. Anaesth pain intensive care 2021;25(2):206-211. DOI: 10.35975/apic.v25i2.1467

Received: 19 November 2020, Reviewed: 22 December 2020, Accepted: 12 January 2021

\section{Introduction}

Congenital diaphragmatic hernias $(\mathrm{CDH})$ are uncommon abnormalities, with Morgagni hernia being the less common, occurring in $1.5 \%$ to $6 \%$ of cases. ${ }^{1}$ Adult presentation is rare. ${ }^{2}$ In $90 \%$ case it is seen on the right side and $10 \%$ cases are either left-sided or bilateral. Morgagni hernia usually contains the transverse colon, omentum, liver and sometimes stomach or small bowel. ${ }^{3}$ Diagnosis is based on clinical and radiological evaluation and computed tomography (CT) is the most sensitive exam. ${ }^{2}$ Herniation of abdominal viscera in the thoracic cavity through the foramen of Morgagni may produce severe cardiopulmonary or gastrointestinal complications. Its surgical treatment is mandatory ${ }^{2}$ and a challenge for the anesthesiologist, mainly due to the wide 
hemodynamic changes and ventilatory complications during the procedure.

Esophageal Doppler (ED) is a noninvasive, inexpensive and easy ${ }^{4}$ hemodynamic monitoring system, with high validity and clinical concordance, ${ }^{5}$ that allows continuous estimation of corrected flow time, stroke volume, and cardiac output ${ }^{1}$ during surgery, facilitating optimized real-time fluid resuscitation, and output management. ${ }^{3}$ The pulmonary artery catheter has been deemed too invasive for the types of surgery being undertaken. ${ }^{6}$

In this case report, we present the successful anesthetic management of a late onset Morgagni hernia repair, using the ED, in a 70-year-old female patient to stress the usefulness of this medium for the anesthesiologists.

\section{Case report}

A 70-y old woman, ASA grade 3, with arterial hypertension and heart failure but preserved ejection fraction (New York Heart Association II/III), on amlodipine $5 \mathrm{mg}$, ramipril $5 \mathrm{mg}$ and furosemide 60 $\mathrm{mg} /$ day, presented with complaints of dyspnea and chronic chest pain, and sought medical observation due to worsening symptoms. Chest $\mathrm{x}$-ray showed opacification of $2 / 3$ of the left and half of the right

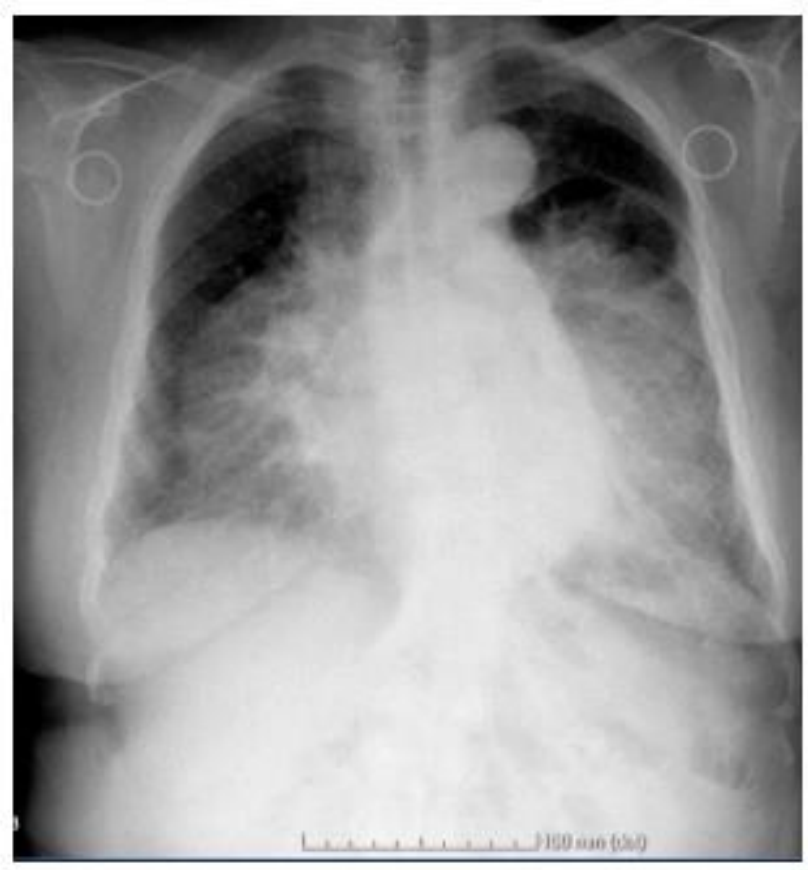

Figure 1: Preoperative chest $\mathrm{X}$-ray hemithorax (Figure 1) and the presence of intrathoracic intestinal loop (Figure 2).

A thoracoabdominal CT scan confirmed the presence of large-sized Morgagni hernia, with colon herniation to the thoracic cavity, compromise of both hemithoraces, and compression of the cardiac chambers (Figure 3). She was scheduled for hernia repair.

In the pre-anesthetic evaluation, there was a decrease in the left pulmonary base and cardiac sounds, associated with bowel noises in the left hemithorax. Hypoxemia $\left(\mathrm{pO}_{2} 67.6 \mathrm{mmHg}\right.$ ) was recognized at room air. As it was a chronic hernia, with years of evolution, the risk of developing pulmonary re-expansion edema after surgery was considerable. A double lumen tube was therefore, made available in the operating room, as was a fiberoptic bronchoscope. Aspiration prophylaxis was made with metoclopramide and ranitidine.

The patient was monitored with ASA standard parameters, invasive blood pressure (IBP) in left radial artery, bispectral index (BIS), diuresis, muscle relaxation (train of four) and ED (CardioQ ${ }^{\circledR}$ by Deltex Medical Limited, Chichester, United Kingdom). The patient presented with IBP at 127-135/78-82 $\mathrm{mmHg}$. The ED initial values are displayed in Figure 4.

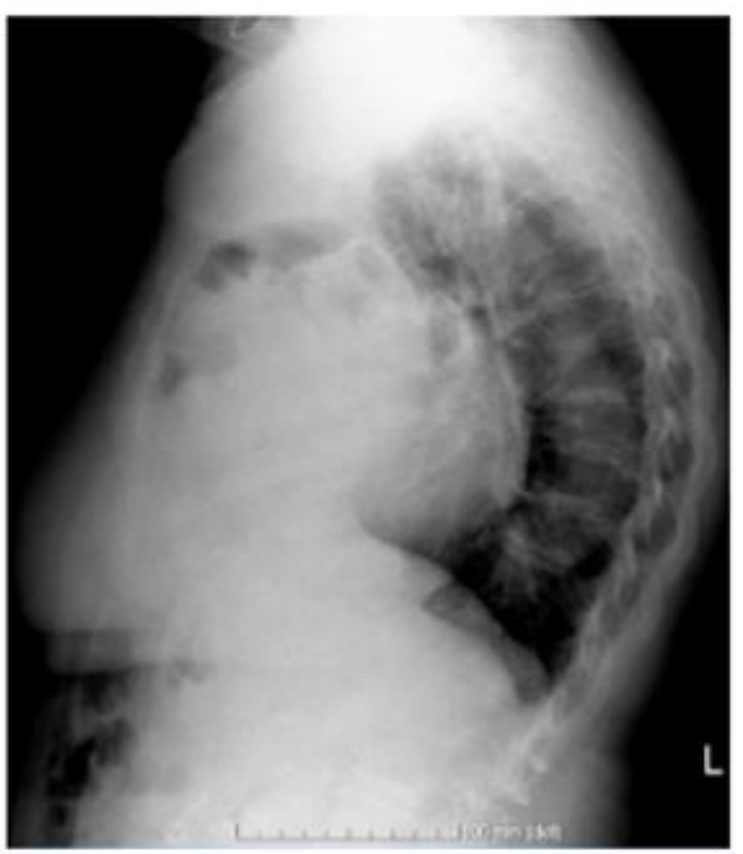

Figure 2: Preoperative chest $\mathrm{X}$-ray left side view 
Balanced general anesthesia was performed. Rapid sequence induction was started with fentanyl $2 \mu \mathrm{g} / \mathrm{kg}$, propofol $2.5 \mathrm{mg} / \mathrm{kg}$ and rocuronium $1.2 \mathrm{mg} / \mathrm{kg}$. Endotracheal intubation was performed at the first attempt without any difficulty with a cuffed tube size
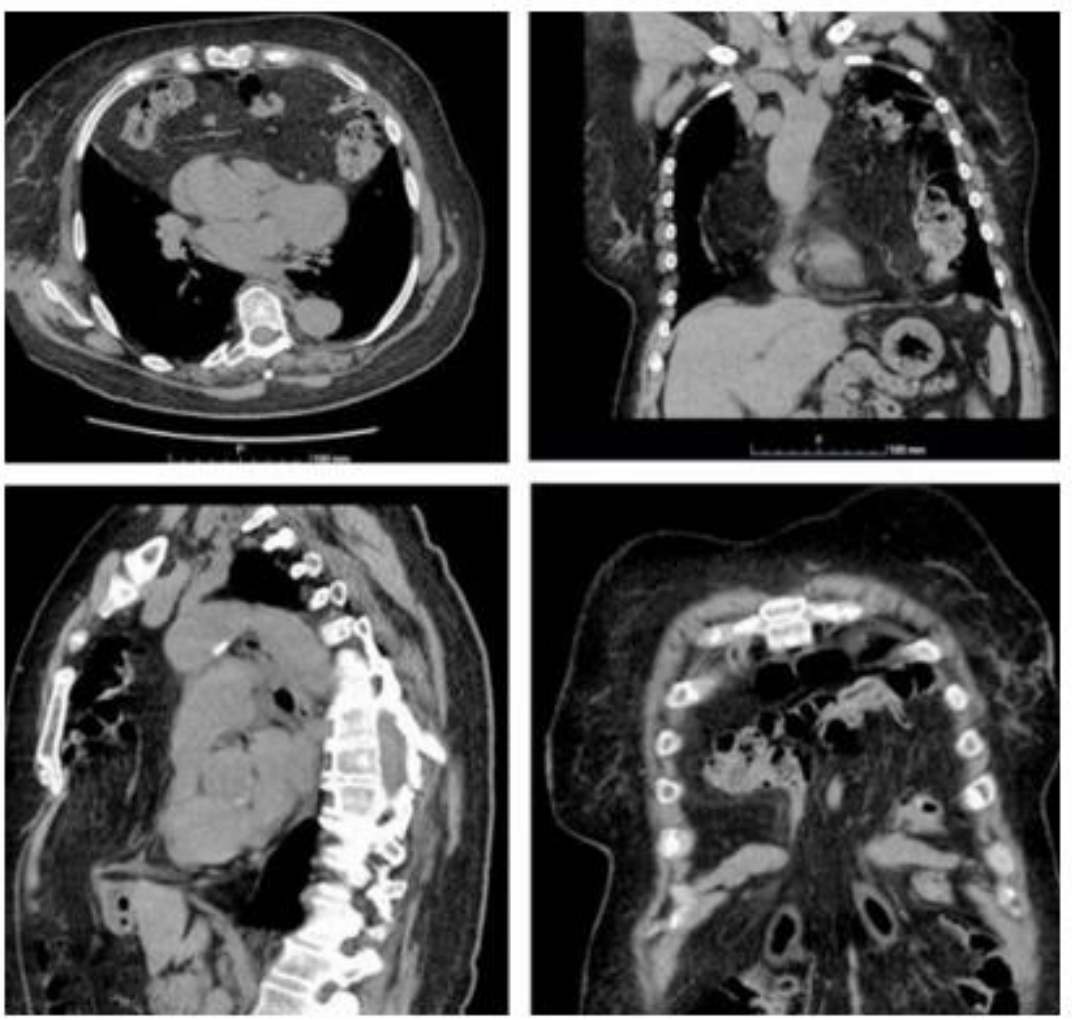

6.5. Sevoflurane was used in a $50 \% \mathrm{O}_{2}+50 \%$ air mixture for maintenance being titrated according to the BIS 40-60.

There was no difficulty in ventilation, using volumecontrolled ventilation with mean pressures $9 \mathrm{cmH}_{2} \mathrm{O}$, compliance $39 \mathrm{ml} / \mathrm{cmH}_{2} \mathrm{O}$, end tidal $\mathrm{CO}_{2}$ values between $35-37 \mathrm{mmHg}$, inspiration/expiration ratio $1: 2$ and positive end-expiratory pressure (PEEP) of $5 \mathrm{cmH}_{2} \mathrm{O}$. Nasogastric tube was introduced.

Laparotomy was performed, with identification of the local herniation, diaphragmatic orifice and herniated content. After removing the herniated content, she had an episode of hypotension; systolic blood pressure (SBP) $75 \mathrm{mmHg}$, mean arterial pressure (MAP) $43 \mathrm{mmHg}$ ), and ED showing a decrease in SV $(45 \mathrm{ml})$ in the CI $\left(1.1 \mathrm{l} / \mathrm{min} / \mathrm{m}^{2}\right)$ and FTc (213 ms), requiring temporary vasopressor support with noradrenaline $(0.05 \mu \mathrm{g} / \mathrm{kg} / \mathrm{min})$ and optimization of fluid therapy with a total of $1100 \mathrm{ml}$ of intraoperative crystalloids. The hemodynamic values were gradually stabilized with the instituted measures (Figure 5).

Figure 3: Thoracoabdominal CT scan

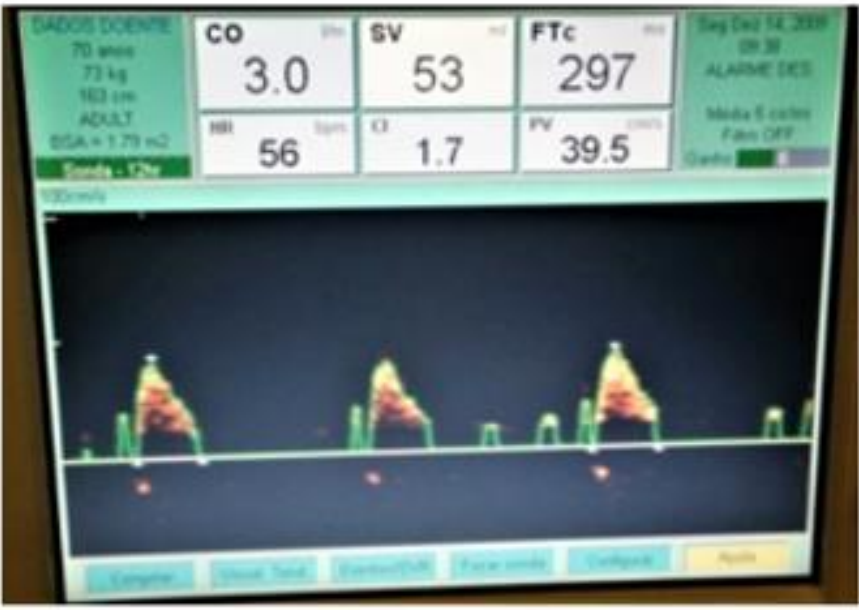

Figure 4 -ED values - beginning of the surgery

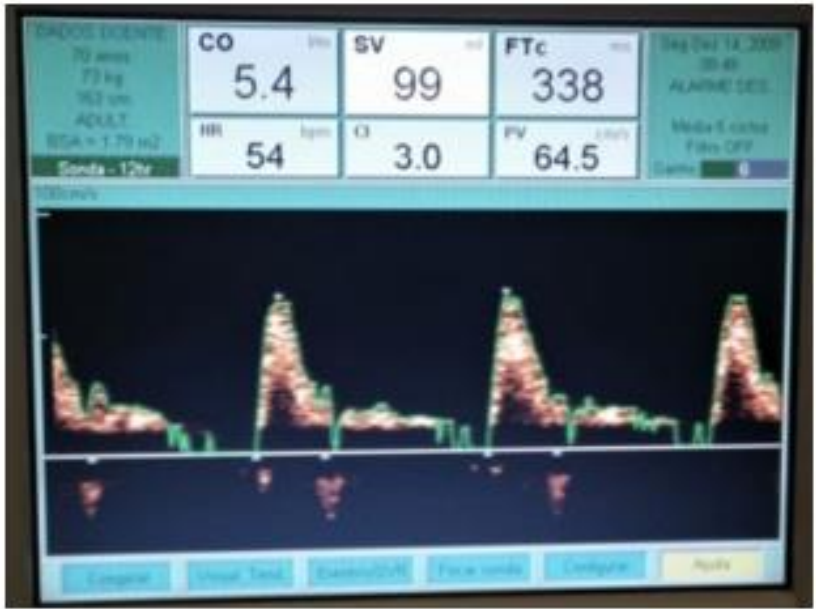

Figure 5: ED values after vasopressor support and optimization of fluid therapy 


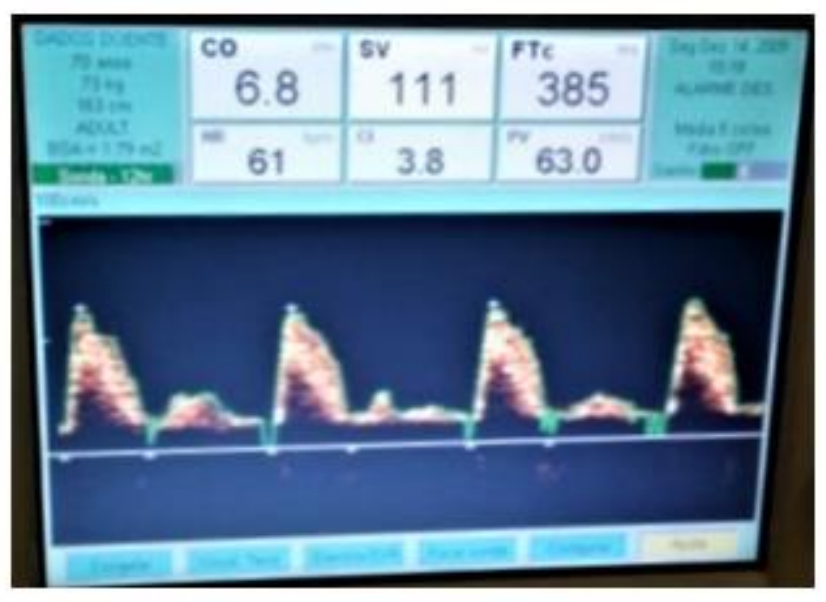

Figure 6: ED values at the end of the intervention

At the end of the intervention, the patient was stable with IBP 108-116/64-72 $\mathrm{mmHg}$ and there was an increase of $109 \%$ in SV, $123 \%$ in CI and 30\% in the FTc (Figure 6) compared to the initial values. There were no intercurrences during pulmonary reexpansion maneuvers. After surgery, neuromuscular blockade was reversed with sugammadex $(2 \mathrm{mg} / \mathrm{kg})$ and extubation done without complications.

There were no complications in the post-anesthetic care unit. She was discharged on the fourth postoperative day, asymptomatic and with radiological normality (Figure 7).

\section{Discussion}

Diaphragm anatomical defect arises during early embryological development (around 10 weeks) due to incomplete closure of the diaphragm or early migration of the mid gut from umbilical celom in to the abdominal cavity before the diaphragm is fully developed. Three common sites of herniation are (A) Posterolaterally through the foramen of Bochdalek (78\%-90\%) (B) esophageal hiatus (14\%-24\%) (C) retrosternally through the foramen of Morgagni $(1.5 \%-6 \%){ }^{1}$ Only 79 cases in a study of 3098 cases of $\mathrm{CDH}$ presented with late onset. ${ }^{1}$ It emphasizes the rarity of our case as our patient was 70 -year-old at the time of presentation.

It arises $90 \%$ on the right side and the remaining cases are either left sided or bilateral. ${ }^{6}$ In this patient, the lesion was in the anteromedial region of the diaphragm and contents of the hernial sac occupied extensively

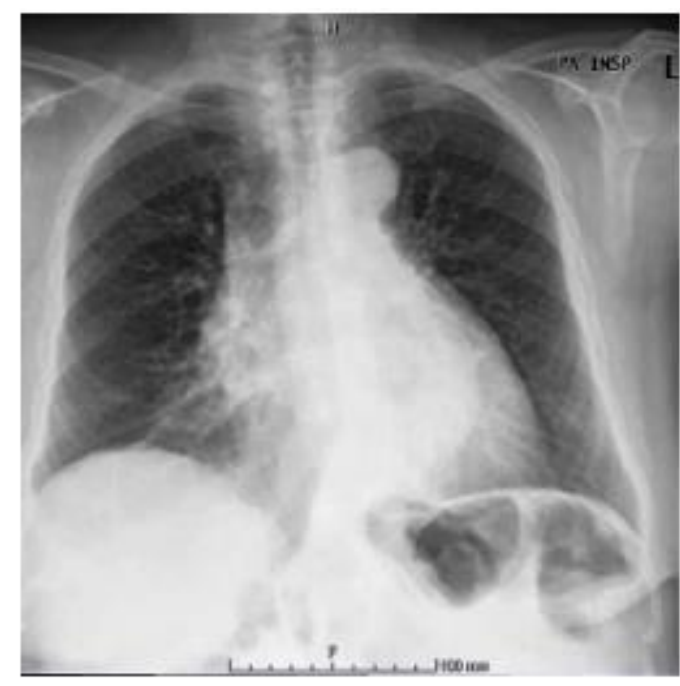

Figure 7: Post-surgical chest $\mathrm{x}$-ray

both hemithoraxes surrounding the pericardial sac. The most common organs to herniate are the colon, omentum, stomach, liver and small intestine, ${ }^{7}$ compromising their functioning and generating cardiorespiratory changes.

It usually manifests during childhood with respiratory symptoms. Late onset $\mathrm{CDH}$ is more difficult to diagnose as sign and symptoms become chronic and vague. Respiratory symptoms such as dyspnea, with or without a cough, are most commonly seen in these patients. ${ }^{8}$ Our patient reported dyspnea, cough and chest pain. Herniated viscera in the thorax may produce a mass effect and can lead to cardiovascular impairment by compression of the heart and mediastinal shift, which can block vena cava, pulmonary veins, impair venous return to heart and cause cardiac output to decrease. ${ }^{1}$

There have been few case reports on anesthetic management of late onset Morgagni hernia in anesthesia literature. Anesthetic management of a patient Morgagni hernia is challenging for anesthesiologists.

A patient with $\mathrm{CDH}$ should be considered full stomach because of possible gastrointestinal obstruction, and, therefore, these patients required aspiration prophylaxis, rapid sequence induction, possibly awake fiberoptic intubation and nasogastric tube. Large gauge intravenous access is necessary to manage any hemodynamic instability. Positive pressure ventilation with potential gastric insufflation and expansion of compressed lung may decrease venous return and 
cardiac output, so is recommended low tidal volume and low airway pressure strategy. Nitrous oxide should be avoided because may also worsen the mass effect. We used sevoflurane for anesthetic maintenance.

Considering the combined mass effect of herniated viscera, and inflated lung may lead to cardiorespiratory impairment, heart filling, inotropic state of the heart and volume content must be closely monitored. Standard ASA monitoring poorly predicts these elements. ${ }^{9}$

The use of ED is of great importance in this context. Literature suggests that customized fluid therapy, guided by cardiac index inferred by ED improves clinical outcomes compared to traditional liberal administration, facilitates optimized vascular filling, minimizes hypovolemia and helps maintain an appropriate global and regional perfusion. ${ }^{10}$

ED provides real-time information on cardiac function and vascular filling. It also measures blood velocity using a low frequency signal beam, directed along the path of blood flowing in the descending aorta, from which, with Doppler principal flow is calculated as a product of velocity and cross-sectional area of the aorta, estimated from the characteristics of the patient.

The Dopplerprobe can be placed through the nose or mouth, and nasal access is more stable and easier to protect, although with an increased risk of trauma and bleeding. The ED screen shows a velocity waveform. Because the velocity of the blood in the aorta is not constant, the graphical representation of the waves assumes a triangular form, with height representing the maximum velocity (MV). A low MV can indicate hypovolemia or increased after-load and a high value indicate after-load decrease.

The flow time is corrected for a heart rate (HR) of 60 beats/min, generating the FTc. Systolic and diastolic time intervals are reduced in tachycardia and prolonged in the bradycardia. The FTc is inversely proportional to the vascular resistance, therefore, in states such as hypovolemia, excess vasopressors or hypothermia the FTc shortens, while in states of low resistance such as sepsis, FTc enlarges.

The typical values for FTc in a healthy adult are 330 $360 \mathrm{~ms}$. Mean acceleration (MA), on the other hand, indicates the average acceleration of the blood from the beginning of systole to the detected MV. The MV expresses the highest blood velocity detected during systole and is used as an indicator of left ventricular contractility. The reduction in MV and MA is related to impaired inotropism resulting, for example, from cardiac depressant drugs, ischemia, or increased afterload. MV decreases approximately $1 \%$ per year till adult life, with normal values of $90-120 \mathrm{~cm} / \mathrm{sec}$ in an adult of $20 \mathrm{y}$ at rest passing to $50-70 \mathrm{~cm} / \mathrm{sec}$ in an adult of $70 \mathrm{y}$ at rest. FTc values less than $330 \mathrm{~ms}$ indicate a poor filling of the ventricle. If a fluid challenge produces an increase in FTc and SV, it can be assumed that this act is effectively increasing the preload in a preload-dependent ventricle. ${ }^{9}$ In this patient, the fluid challenge was insufficient and the parameters pointed to the need for adrenergic support, which was started. The same reasoning was taken in the opposite direction for its suspension, achieving good hemodynamic stability.

Cardiac output ( $\mathrm{CO}$ ) derived from velocimetry, as verified by $E D$, shows a degree of encouraging reliability. ${ }^{9}$ There is a reduction in postoperative complications and hospitalization compared to the conventional clinical evaluation with or without cardiovascular invasive monitoring.

Here, the low SV, CO, CI and FTc registered before the surgical procedure (Figure 4) indicate an increase in afterload and decrease in preload, probably due to the compression of cardiac chambers and large vessels by herniated contents. After removal of the hernia, we observed an increase in the FTc, MV, SV and consequently the $\mathrm{CO}$, corroborating the reasoning.

The esophageal Doppler monitor increases the clinical judgment of the anesthesiologist and allows better hemodynamic management adapted to each patient, in a less invasive and risky way. This technology provides objective information in real time, allowing therapeutic individualization and subsequent decreased morbidity associated with this type of surgery.

Possible complications such as subcutaneous emphysema, pneumothorax, pneumomediastinum and pneumopericardium, should be considered intraoperatively in the presence of crepitation in sternal notch, hypercarbia, hypoxia and increased airway pressure. None of these complications happened in our case. The use of ED allows to identify changes in cardiac flows early in the presence of these complications, such as a decrease in CI, SV or FTc, 
being an important decision support. After extubation was ordered a chest $x$-ray that confirmed the absence of those complications.

A multidisciplinary approach of the anesthetic and surgical teams and a differentiated hemodynamic evaluation was imperative and decisive in this case. The use of ED helped identify these changes early and allowed us to intervene quickly and effectively.

\section{Conflict of interest}

None declare. No external funding was involved in this case.

\section{Authors" contribution}

NB: Concept, drafting and conduction of the work and manuscript editing and final approval of the version to be published

MM, AS: Manuscript editing and revising it critically for important intellectual content

$\mathrm{CC}$ : Manuscript editing and revising it critically for important intellectual content and final approval of the version to be published;

\section{References}

1. Nama RK, Butala BP, Shah V, Patel H. Anesthetic management of Morgagni hernia repair in an elderly woman, Anesth Essays Res. 2015 Sep-Dec;9(3):413416. [PubMed] DOI: $10.4103 / 0259-1162.157467$

2. Razi K, Light D, Horgan L. Emergency repair of Morgagni hernia with partial gastric volvulus: our approach. J Surg Case Rep. 2016 Aug 31;2016(8):rjw151. [PubMed] DOI: 10.1093/jscr/rjw151

3. Beg MH, Rashidi ME, Jain V. Morgagni hernia with down syndrome: a rare association. Indian J Chest Dis Allied Sci. 2010; 52:115-117. [PubMed]

4. Figus A, Wade RG, Oakey S, Ramakrishnan VV. Intraoperative esophageal Doppler hemodynamic monitoring in free perforator flap surgery. Ann Plast Surg. 2013;70(3):301-7. [PubMed] DOI: 10.1097/SAP.0b013e3182306005

5. Ripollés-Melchor J, Casans-Francés R, Espinosa A, Abad-Gurumeta A, Feldheiser A, López-Timoneda F, et al. Goal directed hemodynamic therapy based in esophageal Doppler flow parameters: A systematic review, meta-analysis and trial sequential analysis. Rev Esp Anestesiol Reanim. 2016;63(7):384-405. [PubMed] DOI: 10.1016/j.redar.2015.07.009

6. Green DW. Comparison of cardiac outputs during major surgery using the Deltex CardioQ oesophageal Doppler monitor and the Novametrix-Respironics NICO: A prospective observational study. Int J Surg. 2007 Jun;5(3):176-82. [PubMed] DOI: 10.1016/i.jjsu.2006.05.009

7. Patial T, Negi S, Thakur V. Hernia of morgagni in the elderly: a case report. Cureus. 2017;9(8):e1549. [PubMed] DOI: 10.7759/cureus.1549

8. Reza Afghani, Saeed Amirkhanlu, Hamed Jafarpour, Arash Rezaei Shahmirzadi, Tahere Bakhshi, Morgagni hernia in a 73-year-old woman: a case report. Int J Med Invest. 2017;6(3):99-102. [FreeFullText]

9. Schiraldi R, Calderón L, Maggi G, Brogly N, Guasch E, Gilsanz F. Transoesophageal Doppler-guided fluid management in massive obstetric haemorrhage. Int J Obstet Anesth. 2014 Feb;23(1):71-4. [PubMed] DOI: 10.1016/.ijoa.2013.07.001

10. Srinivasa S, Lemanu D, Singh $P$, Taylor M, Hill A. Systematic review and meta-analysis of oesophageal doppler-guided fluid management in colorectal surgery. Br J Surg. 2013;100(13):1701-1708. [PubMed] DOI: $\underline{10.1002 / \text { bjs. } 9294}$ 